\title{
Longitudinal holograms in terahertz lasers: Electronic tuning and frequency control
}

DOI:

10.1109/IRMMW-THz.2012.6380274

Link to publication record in Manchester Research Explorer

\section{Citation for published version (APA):}

Khairuzzaman, M., Chakraborty, S., Marshall, O., Hsin, C. W., Beere, H., \& Ritchie, D. (2012). Longitudinal holograms in terahertz lasers: Electronic tuning and frequency control. In International Conference on Infrared, Millimeter, and Terahertz Waves, IRMMW-THz|Int. Conf. Infrared, Millim., Terahertz Waves, IRMMW-THz IEEE. https://doi.org/10.1109/IRMMW-THz.2012.6380274

\section{Published in:}

International Conference on Infrared, Millimeter, and Terahertz Waves, IRMMW-THz|Int. Conf. Infrared, Millim., Terahertz Waves, IRMMW-THz

\section{Citing this paper}

Please note that where the full-text provided on Manchester Research Explorer is the Author Accepted Manuscript or Proof version this may differ from the final Published version. If citing, it is advised that you check and use the publisher's definitive version.

\section{General rights}

Copyright and moral rights for the publications made accessible in the Research Explorer are retained by the authors and/or other copyright owners and it is a condition of accessing publications that users recognise and abide by the legal requirements associated with these rights.

\section{Takedown policy}

If you believe that this document breaches copyright please refer to the University of Manchester's Takedown Procedures [http://man.ac.uk/04Y6Bo] or contact uml.scholarlycommunications@manchester.ac.uk providing relevant details, so we can investigate your claim.

\section{OPEN ACCESS}




\title{
Longitudinal Holograms in Terahertz Lasers: Electronic Tuning and Frequency Control
}

\author{
M. Khairuzzaman ${ }^{1}$, S. Chakraborty ${ }^{1}$, O. Marshall ${ }^{1}$, C.-W. Hsin ${ }^{1}$, H. Beere ${ }^{2}$, and D. Ritchie ${ }^{2}$ \\ ${ }^{1}$ School of Electrical and Electronic Engineering, University of Manchester, UK \\ ${ }^{2}$ Cavendish Laboratory, Department of Physics, University of Cambridge, UK \\ Email: s.chakraborty@manchester.ac.uk
}

\begin{abstract}
A longitudinal computer-generated hologram is incorporated into the Fabry-Pérot cavity of a terahertz quantum cascade laser which displays two distinct spectral gain peaks. The combined cavity and hologram feedback mechanisms lead to digitally selective dual-mode emission with a difference frequency which may be electronically tuned from $190 \mathrm{GHz}$ to $267 \mathrm{GHz}$.
\end{abstract}

\section{INTRODUCTION}

$\mathrm{D}$ UAL frequency emission from a semiconductor laser has huge potential benefits for applications such as radio frequency (millimetre and sub-millimetre wave) generation and dense wavelength division multiplexing (DWDM) for high-speed wireless communication systems. Difference frequency generation leading to a beat signal usually requires two independent single-mode lasers, but this approach suffers from temperature drifts and demands precise control of power and polarization of both beams for signal maximization. A single $\mathrm{THz}$ quantum cascade laser (QCL) with two distinct gain peaks, when combined with a simultaneously dual-mode selection mechanism, offers an alternative to multiple laser sources. Multi-peaked THz QCL gain and coarse tuning are possible through active region design. To date, however, achieving user-defined, dual-mode selection with an electronically tuned frequency separation has proven difficult in a single $\mathrm{THz}$ QCL. Here we achieve mode selection with a longitudinal computer-generated hologram (LCGH). These photonic structures can provide flexible, high-resolution digital mode selection at user defined frequencies by breaking a grating periodicity in a deterministic and systematic way ${ }^{1}$.

In this paper, we demonstrate QCLs simultaneously lasing at two digitally selected $\mathrm{THz}$ frequencies by incorporating an LCGH into a pre-existing Fabry-Pérot (FP) QCL cavity (e.g. Fig. 1a). In principle, an LCGH may be designed to give almost any target spectral response. Here, the LCGH was designed to give multiple, finely spaced Bragg resonances (Fig. 1b). This structure, when combined with a two-colour QCL active region with a bias-controllable gain movement, allows simultaneous digital frequency selection on LCGH Bragg resonances (reflection bands). Electronic switching between these digitally selected modes is possible due to both the current (i.e. gain-) dependent longitudinal effective refractive index profile along the FP-LCGH (causing phase shifts between LCGH and non-LCGH waveguide sections), and the bias dependent gain movement ${ }^{1}$.

This work was supported by EPSRC First Grant EP/G064504/1 and partly supported by HMGCC.

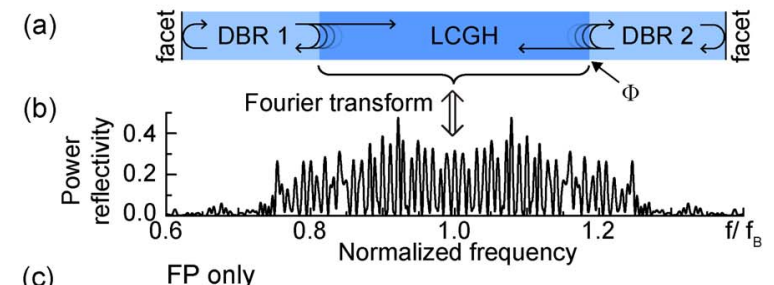

(c)

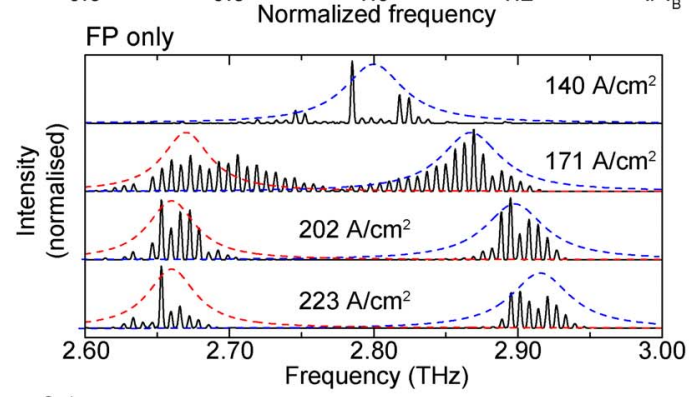

(d)

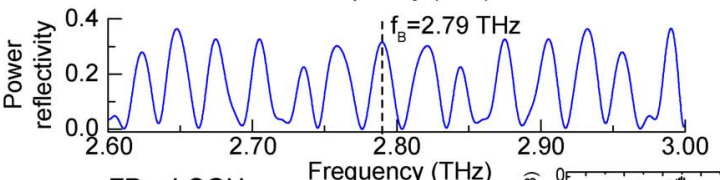

(e)

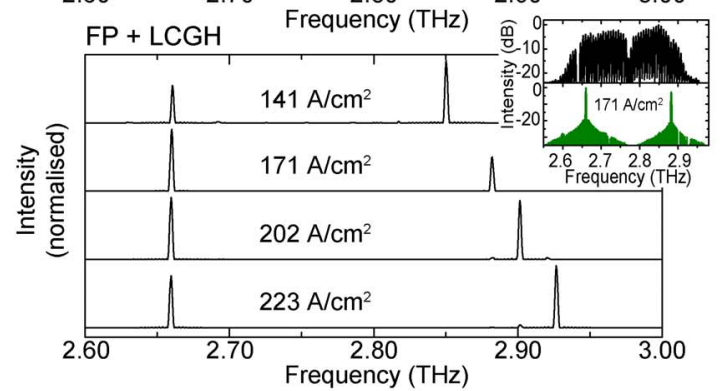

Fig. 1. (a) Schematic of an FP-LCGH cavity with two resultant distributed Bragg reflector (DBR) sub-cavities. (b) LCGH spectral power reflectivity response. (c) Measured FP laser spectra from QCL1 (6.02 mm long, $160 \mu \mathrm{m}$ wide) in pulsed operation at $10 \mathrm{~K}$. Dashed lines are estimates of gain peak positions. (d) Calculated LCGH reflectivity in the spectral region of interest (using $\Lambda=14.60 \mu \mathrm{m}$ and $n_{\text {eff }}=3.68, f_{\mathrm{B}}$ is calculated as $2.789 \mathrm{THz}$ ). (e) QCL1 spectra after introduction of the LCGH. The static mode frequency is $2.660 \mathrm{THz}$, the four switchable 2.849, 2.881, 2.901 and $2.926 \mathrm{THz}$, (average spacing $\sim 26 \mathrm{GHz}$ ). Inset: FP (top) and FP-LCGH (bottom) spectra at $171 \mathrm{~A} / \mathrm{cm}^{2}$, plotted on a logarithmic intensity scale.

\section{FABRICATION AND MEASUREMENT}

Devices were fabricated from a single molecular beam epitaxially grown $\mathrm{GaAs} / \mathrm{Al}_{0.15} \mathrm{Ga}_{0.85}$ wafer, with two heterogeneous bound-to-continuum active regions (ARs), each containing 45 repeat periods, similar to reference 2 . Semi-insulating surface plasmon (SI-SP) ridge waveguides ( $160 \mu \mathrm{m}$ wide) were cleaved to $\sim 6 \mathrm{~mm}$-long FP cavities and fully characterized prior to the incorporation of the LCGH. LCGH gratings were introduced by milling narrow, sub-wavelength slits $(0.6 \mu \mathrm{m}$ by $100 \mu \mathrm{m})$ into the upper metallic layers of the SI-SP waveguides using an FEI Nova 
Nanolab 600 focused ion beam (FIB) system (30 keV Ga ions, $50 \mathrm{~nm}$ nominal spot diameter, $1 \mathrm{nA}$ beam current). These slits followed the LCGH design, with a minimum separation of $\Lambda$, and served to perturb the complex refractive index, $n_{\text {eff, }}$ of the guided mode. The value of $\Lambda$ was adjusted so that the Bragg frequency $f_{\mathrm{B}}\left(f_{\mathrm{B}}=c / 2 n_{\text {eff }} \Lambda, c\right.$ is the speed of light in vacuum) and the QCL gain were appropriately aligned. After milling devices were once again fully characterized.

\section{RESULTS AND DISCUSSION}

Figure 1c shows emission spectra from a $6.02 \mathrm{~mm}$ long, $160 \mu \mathrm{m}$ wide, FP QCL (QCL1). Multiple cavity modes are observed, with a frequency spacing of $\sim 6.50 \mathrm{GHz}$, from which a group refractive index, $n_{g}$, of 3.83 was calculated. The FP modes correspond to two distinct gain peaks, one centered at $\sim 2.66 \mathrm{THz}$, the second tuning with driving currents from $\sim 2.80$ to $2.92 \mathrm{THz}$. In contrast, after LCGH milling (using $\Lambda=14.60 \mu \mathrm{m})$, only two simultaneous modes are observed at almost all driving currents (Fig. 1e). As expected, low frequency mode remains stationary while the high frequency mode switches between four positions under different electrical driving conditions, the tuning resolution dictated by the LCGH reflection bands shown in Fig. 1d. Dual-mode side mode suppression ratios (SMSR) are $\sim 20 \mathrm{~dB}$ (inset Fig. 1).

To ascertain the reproducibility of dual-mode FP-LCGH operation, a second device was chosen (QCL2) with very similar FP performance characteristics to QCL1 ${ }^{3}$. A nominally identical LCGH (i.e. $\Lambda=14.60 \mu \mathrm{m}$ ) was introduced to QCL2. Once again, simultaneous dual-mode lasing was achieved with four possible frequency separations. However, in QCL2 only three high frequency modes were present, though an additional low frequency mode was recorded. Fig. 2a displays the LCGH spectra for QCL1 and QCL2 on a normalized frequency scale. At higher driving currents the dual-mode pairs are closely matched in frequency. Lower driving currents produced lasing pairs with a relative frequency shift of one LCGH band (shaded bars). During FIB milling, neither the facet phase nor the precise value of complex refractive index contrast was controlled. Both affect the relative optical path lengths of the four laser cavity elements (FP resonator, LCGH and two subcavities: DBR1, DBR2 in Fig. 1a) and thereby influence the complex round-trip gain and phase solutions which in turn will cause variation in the spectral position of the output modes. Furthermore, subtle differences in gain peak positions in the two QCLs might also explain lasing in neighboring LCGH bands at low driving currents. Despite difference between the QCLs, all lasing modes do follow the LCGH response, with the average switchable mode spacing dictated by the LCGH resonance separation. Average switchable mode spacings of $0.009 f_{\mathrm{B}}(\sim 26 \mathrm{GHz})$ and $0.008 f_{\mathrm{B}}(\sim 22 \mathrm{GHz})$ are measured for QCL1 and QCL2 respectively, which closely match with the designed value of $0.01 f_{\mathrm{B}}$. Other FP-LCGH QCLs displayed similar dual-mode behaviour ${ }^{3}$.

The simultaneous selection of a robust (QCL1) or switchable (QCL2) low frequency single mode, with a switchable high frequency single mode (for both QCLs), gives a tunable frequency difference, given in Fig. 2b. The electronically controlled difference frequency, $\Delta f$, can be varied from 190 to $267 \mathrm{GHz}$ for QCL1 (blue) and from 202 to $267 \mathrm{GHz}$ for QCL2 (red). Figure 2b also displays the driving current spans over which the dual mode pairs are observed. It should be noted that one can target a desired $\Delta f$ by tailoring the LCGH design to vary the Bragg resonance separation. This freedom of choice is one of the novelties of LCGH design.
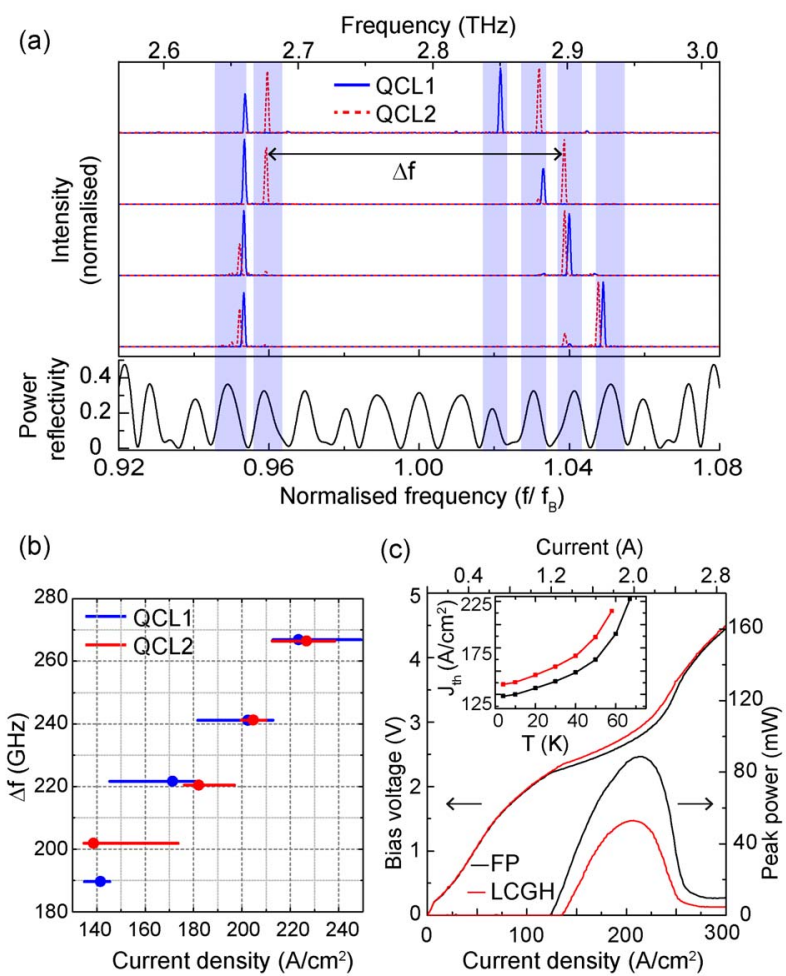

Fig. 2. (a) Measured FP-LCGH spectra on a normalised frequency scale (top), correlated with the LCGH reflectivity response (bottom). (b) The frequency separation, $\Delta \mathrm{f}$, between simultaneously lasing modes as a function of current density for both devices. Lines indicate operational ranges of lasing modes, circles the point of maximum SMSR for each mode pair. (c) Electrical and optical performance of QCL1 before and after LCGH introduction. Inset: threshold current density, $J_{\mathrm{th}}$, as a function of operating temperature.

Fig. 2c displays the electrical (V-J) and optical power (L-J) performance of QCL1 before and after FIB milling. The LCGH grating did not significantly modify the form of the $\mathrm{V}-\mathrm{J}$, though elevated waveguide losses raised the threshold current by $\sim 8 \%$ with a commensurate drop in the maximum operating temperature (inset). Around $60 \%$ of the original $\mathrm{THz}$ power $(53 \mathrm{~mW})$ was retained after LCGH milling.

\section{CONCLUSION}

Combining a longitudinal hologram with a two-colour gain medium, purely electronic tunable simultaneous dual-mode lasing in a single-section THz QCL was achieved. SMSR of better than $\sim 20 \mathrm{~dB}$ for both modes was maintained over a wide tuning current range. This stable lasing behavior is suitable for various applications where dual frequency operation is needed. Furthermore, the demonstration of such tunable frequency differences opens new possibilities for the generation and measurement of tunable beat signals and thereby becomes a promising alternative to existing difference frequency generation techniques.

\section{REFERENCES}

[1] S. Chakraborty, O. Marshall, M. Khairuzzaman, C.-W. Hsin, H. E. Beere, and D. A. Ritchie, "Longitudinal Computer Generated Holograms for Digital Frequency Control in Electronically Tunable Terahertz Lasers", http://arxiv.org/abs/1205.3042

[2] J. Freeman, J. Madeo, A. Brewer, S. Dhillon, O. P. Marshall, N. Jukam, D. Oustinov, J. Tignon, H.E. Beere, D. A. Ritchie, "Dual wavelength emission from a terahertz quantum cascade laser", Appl. Phys. Lett., 96, 051120 (2010).

[3] M. Khairuzzaman, S. Chakraborty, O. Marshall, H. Beere, D. Ritchie, unpublished. 
